\title{
STUdied ON A MULTICLADDEd ERBIUM DOPED DISPERSION COMPENSATING FibER AMPLIFIER
}

\author{
Mithun De ${ }^{1}$, Indranil Mondal ${ }^{2}$ and Amit Kumar Das ${ }^{3}$ \\ ${ }^{1}$ Department of Applied Science and Humanities, Global Institute of \\ Management and Technology, Krishnagar,Nadia. \\ \{mithun.de9, indranil.physics, amitbsp28\} egmail.com
}

\begin{abstract}
Erbium doped fiber amplifiers (EDFAs) are the essential components of a highly efficient, long distance optical data link.Their design has been refined to give better performance parameters. A novel design approach for erbium-doped fiber amplifiers is proposed based on Matlab and Fortran 77 Programming.In this paper, a combination of fiber intensity distribution, pump and signal power, optimum length and maximum gain are taken into account as objective function and the results are presented for different core radius, fiber length, pump power and signal power. Dispersion compensating fibers (DCFs) which possess negative dispersion coefficient equal to or greater than this $17 \mathrm{ps} / \mathrm{km}-\mathrm{nm}$ can be used to overcome this drawback. In order to upgrade the present long haul fiber optic communication system, comprising of CSFs, a combination of EDFAs and DCFs would be the most feasible choice to compensate the dispersion as well as the loss.
\end{abstract}

\section{KEYWORDS}

Dispersion Compensating Fiber, Dispersion Shifted Fiber, Erbium Doped Fiber Amplifier, Scalar Wave equation of Core profile, Pump power and Signal Power.

\section{INTRODUCTION}

Tremendous technical advancements to develop the fiber optic communication system are in progress to fulfill the great demand for increasing capacity of transmission of information. The long haul fiber optics communication system is mainly comprised of conventional single mode fibers (CSF). The Si based CSFs have low absorption window in the operating wavelength of $700 \mathrm{~nm}$ to $1700 \mathrm{~nm}$. If the attenuation loss is considered, then the resultant loss spectrum shows two low loss window at the wavelength of $1300 \mathrm{~nm}(\operatorname{loss} 0.4 \mathrm{~dB} / \mathrm{km})$ and $1550 \mathrm{~nm}(\operatorname{loss} 0.2 \mathrm{db} / \mathrm{km})$ [1]. Thus the conventional optical fiber system operating at $1300 \mathrm{~nm}$ would be limited by the loss of the fiber, though the dispersion is almost zero at this wavelength. To exploit the lowest loss window of silica based fibers at $1550 \mathrm{~nm}$, if the zero dispersion wavelength is shifted to $1550 \mathrm{~nm}$; one can achieve minimum loss as well as zero dispersion simultaneously. This type of system would consequently lead to a very high band width system with repeater less transmission. The shift of zero dispersion wave length can be accomplished by changing some of the fiber parameters. So the fiber now has zero dispersion at $1550 \mathrm{~nm}$ wavelength, which is also the lowest loss window of Si based fiber. This type of fiber is called dispersion shifted fiber (DSF). The fundamental limit of fiber loss in DSFs could be eliminated by the use of Erbium doped fiber amplifiers (EDFAs) operating at $1550 \mathrm{~nm}$, which increases the spectral efficiency to achieve a greater transmission capability. The choice of DSFs for replacement of already installed network

Rupak Bhattacharyya et al. (Eds) : ACER 2013, 
is not commercially promising due to strong pressure to upgrade these links in making them compatible with DSF and EDFA combination. In the absence of a suitable commercially available $1300 \mathrm{~nm}$ to $1550 \mathrm{~nm}$ of the existing link, so that EDFAs could be employed [2]. The main disadvantage of this approach is in paying the penalty in terms of large chromatic dispersion, which is $(\sim 17 \mathrm{ps} / \mathrm{km}-\mathrm{nm})$ at $1550 \mathrm{~nm}$. Nowadays the information carrying capacity can be increased enormously by using Wavelength Division Multiplexing (WDM) system. WDM system has been widely introduced for large capacity of transmission. Transmission performances of the present long haul optical transmission system using different signals can be limited by the presence of dispersion, non-linearity and noise [1]. Presently optical fibers are being used around the zero dispersion wavelengths to achieve maximum band width. The system is operated at the zero dispersion wave length.

At the same time it is well known that the fundamental limits of fiber loss in single mode fibers can be eliminated by introduction of erbium doped fiber amplifier(EDFA) [1] operating at 1550 $\mathrm{nm}$ to achieve greater transmission distance. The recent development of practical erbium doped fiber amplifier (EDFAs) has greatly reduced the effect of attenuation which acts as a critical design issue in light wave communication. The EDFAs have characteristics of high gain, band width, low loss and high efficiencies. Several research works on EDFA shows that they can be used in wavelength division multiplexing system (WDM) finding a variety of application in travelling wave fiber amplifier, nonlinear optical devices [3]. In the design of EDFAs it is necessary to determine amplifier parameter such as optical fiber length and the optical pumping wavelength to obtain a maximum gain or band width of the EDFA [4].

For low pump powers, although the $\mathrm{Er}^{3+}$ ions are getting excited to the $\mathrm{E}_{2}$ level, population inversion may not exist because of spontaneous emission. Thus in such a case the signal beam at $1550 \mathrm{~nm}$ will get attenuated (due to the absorption from $\mathrm{E}_{1} \mathrm{toE}_{2}$ ) rather than being amplified. As pump power increases, the rate of excitation increases and at some power level one can achieve population inversion between $E_{2}$ to $E_{1}$ and in such a situation the signal around $1550 \mathrm{~nm}$ will get amplified rather than absorbed. This is the basic principle behind optical amplification by $\mathrm{Er}^{3+}$ doped fiber. The development of erbium doped glass fiber has a major impact on the research in the area of active fiber technology when fiber is operated in the $1.55 \mu \mathrm{m}$ wavelength region. After the pioneering work on fiber laser, research on rare earth doped fiber, the research on this area remained stagnant until the demonstration of high gain erbium doped amplifier [3-4] and their use in light transmission system [5]. These active fibers are finding diverse application in optical amplifiers, laser switches, and variety of nonlinear devices. Some of the basic notable results achieve with doped fiber amplifiers are $51 \mathrm{~dB}$ gain, $100 \mathrm{mw}$ saturation output power and $3 \mathrm{~dB}$ noise figure. Such high performance amplifier may enhance the light wave system and have already been used to achieve numerous transmission records. Erbium doped fiber also have a major impact in fiber laser and super fluorescent light source. Here recent achievement includes $71 \%$ pumping efficiency, $60 \mathrm{kHz}$ laser line width and passive mode locking of a fiber laser. Several experiment on optical switching and optical nonlinearities performed to highlight the potential of the rare earth doped fiber in photonic system or instrumentation. Many works also have been done in modeling and design of Erbium doped fiber amplifier [5-7].

In spite of the many advantage there have some drawback of EDFA. These are limited to operating wavelength zone of 1530-1570 nm only. For $1300 \mathrm{~nm}$ system efforts are under way to develop amplifiers based on other rare earth element such as praseodymium.

The present work is mainly concerned with the design of a parabolic index Erbium doped Erbium doped dispersion compensating fiber(Er-DCF). Here we design the fiber by choosing the proper V-parameter, core radius (a), $n_{1} \& n_{2}$ represents the refractive index at the centre core \& the clad and $n_{3}, n_{4}$ are the refractive indices of the first depressed region $\&$ second depressed 
region respectively. The performance of the designed Er-DCF has been studied in terms of gain variation, signal power and pump power variation with length of the fiber. We have studied maximum gain variation and optimum length variation with input pump power for fibers with different signal power has been studied also.

A multicladded single mode optical fiber is chosen in this work to act as an Erbium doped fiber amplifier. The refractive index profile of the chosen fiber is shown figure.

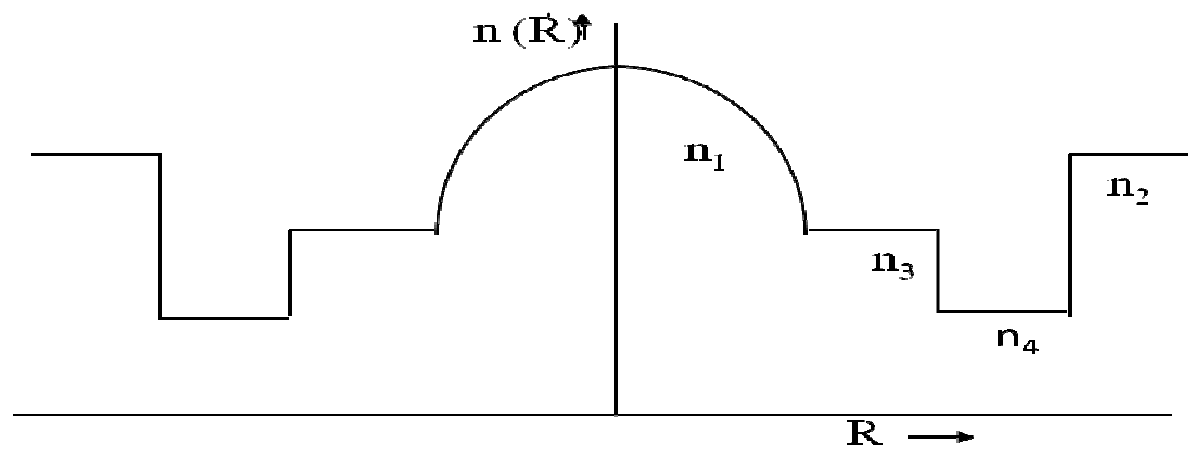

Fig.1: The refractive index profile for a single mode Fiber

The refractive index profile can be described as

$$
\begin{aligned}
n^{2}(\mathrm{R})=n_{1}^{2}\left(1-2 \Delta R^{2}\right) & & ; 0<\mathrm{R} \leq 1 & \\
& =n_{3}^{2}=n_{1}^{2}\left(1-2 \rho_{1} \Delta\right) & & ; 1<\mathrm{R} \leq C_{1} \\
& =n_{4}^{2}=n_{1}^{2}\left(1-2 \rho_{2} \Delta\right) & & ; C_{1}<\mathrm{R} \leq C_{2} \\
& =n_{2}^{2}=n_{1}^{2}(1-2 \Delta) & & ; \mathrm{R} \geq C_{2}
\end{aligned}
$$

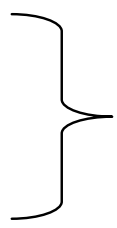

Where, $\mathrm{R}=\mathrm{r} / \mathrm{a}$, a being the core radius of the fiber, $\mathrm{r}$ is the radial coordinate from the centre, $n_{1} \& n_{2}$ represents the maximum refractive index at the centre core $(0<\mathrm{R} \leq 1) \&$ the clad $\left(\mathrm{R}>C_{2}\right)$ and $n_{3}, n_{4}$ are the refractive indices of the first depressed region $\left(1<\mathrm{R} \leq C_{1}\right) \&$ second depressed regions $\left(C_{1}<\mathrm{R} \leq C_{2}\right)$ respectively. The different parameters used here are defined as follows:

The relative refractive index difference $\Delta=\frac{n_{1}^{2}-n_{2}^{2}}{2 n_{1}^{2}}$ and the relative depression depth parameters are given by

$\rho_{1}=\frac{n_{1}^{2}-n_{3}^{2}}{n_{1}^{2}-n_{2}^{2}} \& \rho_{2}=\frac{n_{1}^{2}-n_{4}^{2}}{n_{1}^{2}-n_{2}^{2}}$

The normalized radial coordinate of central parabolic profile, first depressed region and second depressed region are $1, C_{1}, C_{2}$ respectively and the corresponding width of first depressed region $\left(\mathrm{w}_{1}\right) \&$ second depressed region $\left(\mathrm{w}_{2}\right)$ are defined as $\mathrm{w}_{1}=\mathrm{c}_{1}-1 \& \mathrm{w}_{2}=\mathrm{c}_{2}-\mathrm{c}_{1}$.

\section{CHARACTERISTICS OF DISPERSION COMPENSATED FIBER}

Compensation of dispersion at a wavelength around $1550 \mathrm{~nm}$ in a $1310 \mathrm{~nm}$ optimized single mode fiber can be achieved by specially designed fibers whose dispersion coefficient (D) is negative and large at $1510 \mathrm{~nm}$. This type of fiber is known as dispersion compensating fibers (DCF).

Since the DCF has to be added on to an existing fiber optic limit, it would increase the total loss of the system and, hence, would pose problem in detection at the end. The length of the DCF required for compensation can be reduced by having fibers with very large negative dispersion 
coefficients. Thus, there has been considerable research effort to achieved DCFs with very large (negative) dispersion coefficients.

The dispersion compensating fibers are characterized by the parameter, namely compensating ratio (CSR) [8]. The CSR is defined as the ratio of length of the DCF ( $l_{c}$ ) to the length of the $\operatorname{CSF}\left(l_{s}\right)$.

$$
\mathrm{CSR}=\frac{l_{c}}{l_{s}}
$$

For a long haul fiber optic link consisting of a CSF and a DCF, the total dispersion $D_{t}$ due to the propagation along the length $\left(l_{s}\right)$ of a CSF or NZDCF and a length $\left(l_{c}\right)$ of a DCF, is given by [8-9]

$$
D_{t}=D_{s} l_{s}+D_{c} l_{c}
$$

If the total compensation of the dispersion is required, the length of $l_{c}$ is so chosen that

$$
D_{t}=0 \text {.i.e } l_{c}=\frac{D_{s}}{\left|D_{c}\right|} l_{s}
$$

Where, $D_{c}$ is the dispersion coefficient of the DCF in ps $/ \mathrm{km}-\mathrm{nm}$ and $D_{s}$ is that of CSF. The total attenuation of the link $\alpha$ (in $\mathrm{dB}$ ) due to the length lc of DCF is given by [8-9]:

$$
\begin{array}{r}
\alpha=\alpha_{s} l_{s}+\alpha_{c} l_{c} \\
\text { Or, } \quad \alpha=\left(\alpha_{s}+\alpha_{c} \frac{D s}{|D c|}\right) 1_{s}
\end{array}
$$

Where $\alpha_{\mathrm{s}}$ and $\alpha_{\mathrm{c}}$ are the losses of a CSF and a DCF, respectively, in $\mathrm{dB} / \mathrm{km}$. From eqn. (2.4.5), it is seen that the total link attenuation is increased by inserting DCFs. Hence the added attenuation must be compensated with additional gain of the amplifiers. The DCFs should be optimized to minimize the total loss of the fiber.

\subsection{Amplifier Modeling}

We consider an erbium doped single mode silica fiber in which the core is doped with $\mathrm{Er}^{+3}$ ions with ion density of $\mathrm{N}_{\mathrm{t}}(\mathrm{r})$. The pump and signal wavelengths are $980 \mathrm{~nm}$ and $1550 \mathrm{nmrespectively.}$ Here all the parameters such as intensities, population densities of each level are considered as independent of azimuthal angle $\Phi$ due to its axial symmetry. Let $\mathrm{N}_{1}(\mathrm{r}, \mathrm{z})$ and $\mathrm{N}_{2}(\mathrm{r}, \mathrm{z})$ be the population densities of $\mathrm{Er}^{3+}$ in the ground state, $\mathrm{E}_{1}\left(\mathrm{I}^{4}{ }_{15 / 2}\right)$ and the upper amplified state $\mathrm{E}_{2}\left(\mathrm{I}^{4}{ }_{13 / 2}\right)$ respectively. Here $\mathrm{r}$ represents the cylindrical radial coordinates and $\mathrm{z}$ is propagation length along the $\mathrm{z}$ axis. The total ion density is considered as

$$
\mathrm{N}_{1}(\mathrm{r}, \mathrm{z})+\mathrm{N}_{2}(\mathrm{r}, \mathrm{z})=\mathrm{N}_{\mathrm{t}}(\mathrm{r})
$$

Let $I_{p}$ and $I_{s}$ represents the intensity distribution of the pump and signal beams. . Let $\sigma_{\mathrm{pa}}, \sigma_{\mathrm{sa}} \& \sigma_{\mathrm{se}}$ be the absorption cross-section at the pump, signal, \& emission cross section at the signalrespectively. We may then write for the rate of change of population of the ground level, E1 as

$$
\frac{d N_{1}}{d t}=-N_{1} \frac{\sigma_{p a} I_{P}}{h v_{p}}-\frac{I_{s} \sigma_{s a}}{h v_{s}} N_{1}+\frac{I_{s} \sigma_{s e}}{h v_{s}} N_{2}+\frac{N_{2}}{t_{s p}}
$$

Where $N_{1} \frac{\sigma_{p a} I_{P}}{h v_{p}}=$ number of absorptions (per unit time per unit volume) from level $\mathrm{E}_{1}$ to the upper pump level $\mathrm{E}_{3}$ due to the pump at $v_{\mathrm{p}}$. 
$\frac{I_{s} \sigma_{s a}}{h v_{s}} N_{1}=$ number of absorptions (per unit time per unit volume) from level $\mathrm{E}_{1}$ to the upper level $\mathrm{E}_{2}$ due to the signal at $v_{\mathrm{s}}$.

$\frac{N_{2}}{t_{s p}}=$ number of spontaneous emissions (per unit time per unit volume) from level $\mathrm{E}_{2}$ to the $\mathrm{E}_{1}$ Since, $\quad \sigma_{\mathrm{se}} / \sigma_{\mathrm{sa}}=\eta$

So we can write,

At steady state $\quad \frac{d N_{1}}{d t}=0$

$$
\frac{d N_{1}}{d t}=-N_{1} \frac{\sigma_{p a} I_{P}}{h v_{p}}+\frac{I_{s} \sigma_{s a}}{h v_{s}}\left(\eta N_{2}-N_{1}\right)+\frac{N_{2}}{t_{s p}}
$$

$$
\begin{aligned}
& \text { or, } N_{1} \frac{\sigma_{p a} I_{P}}{h v_{p}}+\frac{I_{s} \sigma_{s a}}{h v_{s}} N_{1}=\frac{I_{s} \sigma_{s a}}{h v_{s}} \eta N_{2}+\frac{N_{2}}{t_{s p}} \\
& \frac{N_{1}}{N_{2}}=\frac{\frac{\sigma_{s a}}{h v_{s}} I_{s} \eta+\frac{1}{t_{s p}}}{\frac{\sigma_{p a} I_{p}}{h v_{p}}+\left(\frac{\sigma_{s a} I_{s}}{h v_{s}}\right)} \\
& \frac{N_{2}}{N_{1}}=\frac{\frac{\sigma_{p a I_{p}}}{h v_{p}}+\left(\frac{\sigma_{s a} I_{s}}{h v_{s}}\right)}{\frac{\sigma_{s a}}{h v_{s}} I_{s} \eta+\frac{1}{t_{s p}}} \\
& \frac{N_{2}}{N_{1}}=\frac{\frac{\sigma_{p a} I_{p}}{h v_{p}} t_{s p}+t_{s p}\left(\frac{\sigma_{s a} I_{s}}{h v_{s}}\right)}{\left(\sigma_{s a} \eta I_{s}+1\right)} \\
& \frac{N_{2}}{N_{1}}=\frac{\left\{\frac{\frac{I_{p}}{h v_{p}}}{\sigma_{p a} t_{s p}}+\frac{\frac{I_{s}}{h v_{s}[1+\eta]}}{\sigma_{s a} t_{s p[1+\eta]}}\right\}}{\left\{1+\frac{\frac{I_{s} \eta}{h v_{s}[1+\eta]}}{t_{s p} \sigma_{s a}[1+\eta]}\right\}} \\
& \frac{N_{2}}{N_{1}}=\frac{\tilde{\mathrm{I}}_{P}+\frac{\tilde{\mathrm{I}}_{S}}{[1+\eta]}}{1+\frac{\tilde{\mathrm{I}}_{S} \eta}{[1+\eta]}}
\end{aligned}
$$

Where, $\tilde{I}_{P}=\frac{\frac{I_{p}}{h v_{p}}}{\sigma_{p a} t_{s p}}=\frac{I_{p}}{I_{p o}}$

$$
\tilde{\mathrm{I}}_{S}=\frac{I_{S} \sigma_{s a} t_{s p}[1+\eta]}{h v_{s}}=\frac{I_{S}}{I_{s o}}
$$

Using the result we get, $N_{t}=N_{1}\left(1-\frac{1+\frac{\eta \tilde{I}_{s}}{[1+\eta]}}{1+\tilde{I}_{s}+\tilde{I}_{p}}\right)$

Solving we get, $N_{1}=N_{t}\left(\frac{1+\frac{\eta \tilde{I}_{s}}{[1+\eta]}}{1+\tilde{I}_{s}+\tilde{I}_{p}}\right)$

$$
N_{2}=N_{t}\left(\frac{\tilde{\mathrm{I}}_{p}+\frac{\tilde{\mathrm{I}}_{s}}{1+\eta}}{1+\tilde{\mathrm{I}}_{s}+\tilde{\mathrm{I}}_{p}}\right)
$$

Where $N_{1} \& N_{2}$ represents steady state population of energy level $E_{1} \& E_{2}$ respectively.The condition of amplification has $\left(\eta \mathrm{N}_{2}-\mathrm{N}_{1}\right)>0$ 
Using the above equation we get,

$\eta \mathrm{N}_{2}-\mathrm{N}_{1}=\frac{\tilde{\mathrm{I}}_{p}(r, z) \eta-1}{1+\tilde{\mathrm{I}}_{S}+\tilde{\mathrm{I}}_{p}} \mathrm{~N}_{\mathrm{t}}$

Hence for amplification (at a particular value of $\mathrm{r} \& \mathrm{z}$ ) we must have, $\tilde{I}_{p}(r, z)>\frac{1}{\eta}$

Or, $\mathrm{I}_{\mathrm{p}}(\mathrm{r}, \mathrm{z})>\mathrm{I}_{\mathrm{pt}}=\frac{1}{\eta} I_{p o}$

Where $\mathrm{I}_{\mathrm{pt}}$ is known as the threshold pump intensity. Thus a minimum pump intensity is needed at any value of $(r, z)$ to achieve amplification.

\subsection{Variation of pump and signal power intensity with length}

Since pump wave at frequency $v_{\mathrm{p}}$ corresponds to transitions between $\mathrm{E}_{1}$ and $\mathrm{E}_{3}$ and since population of $\mathrm{E}_{3}$ level is negligible, we can write for the rate of change of pump intensity $\frac{d I p}{d z}=-\sigma_{\mathrm{pa}} \mathrm{N}_{1}(\mathrm{r}, \mathrm{z}) \mathrm{I}_{\mathrm{p}}(\mathrm{r}, \mathrm{z})$

Similarly the change in signal intensity with z described by, $\frac{d I s}{d z}=\sigma_{\mathrm{sa}}\left(\eta \mathrm{N}_{2}-\mathrm{N}_{1}\right) \mathrm{I}_{\mathrm{s}}(\mathrm{r}, \mathrm{z})$

In case of optical fiber the amplification can be described in terms of the signal and pump power rather than in terms of intensities. Since the propagating mode at the pump and signal wavelength are characterized by transverse intensity profile that is, $I_{p}$ and $I_{s}$ which are also the function of transverse coordinate $r$. If we assume that both the pump and signal radiation exists in the fundamental mode of the doped fiber, then we get

$$
\frac{d p_{p}}{d z}=-2 \Pi \sigma_{p a} N_{0} \int_{0}^{b} \frac{1+\left(\frac{\eta}{1+\eta}\right)\left(\frac{P_{s}(z) f s(r)}{I_{s o}}\right)}{1+\frac{P p(z) f p(r)}{I_{p o}}+\frac{P_{S}(z) f s(r)}{I_{s o}}} P p(z) f p(r) r d r
$$

Similarly for the signal power we have,

$$
\frac{d p_{s}}{d z}=2 \Pi \sigma_{s a} N_{0} \int_{0}^{b} \frac{\left(\eta \frac{P_{p}(z) f_{p}(r)}{I_{p o}}\right)-1}{1+\frac{P p(z) f p(r)}{I_{p o}}+\frac{P_{s}(z) f s(r)}{I_{s o}}} P s(z) f p(r) r d r
$$

Where $\mathrm{P}_{\mathrm{p}}(\mathrm{z})$ and $\mathrm{P}_{\mathrm{s}}(\mathrm{z})$ are $\mathrm{z}$ dependent powers at the pump and signal wavelengths and the quantities $f_{p}(r)$ and $f_{s}(r)$ represents the transverse dependence of the modal intensity patterns of the pump and signal waves respectively.

As the pump and signal intensity depends on fiber length, the gain will also depend on fiber length. Here gain increases with fiber length, so the fiber can act as an amplifier. For every input pump power level, there is an optimum length of the fiber for achieving maximum gain. This behavior can be easily understood from the fact that, as the pump propagates through the fiber, it gets absorbed and the pump power level keeps falling monotonically with $z$. Thus, the inversion keeps reducing with $\mathrm{z}$, which in turn reduces the gain at any value of $\mathrm{z}$. At some $\mathrm{z}$ value the pump power drops below the critical value, wherein the fiber becomes attenuating rather than 
amplifying. Any fiber length beyond this point reabsorbs the amplified signal, thus reducing the gain.

\section{RESULT AND DISCUSSION}

In order to design the erbium doped dispersion compensating fiber (ErDCF) having multicladded refractive index profile with total dispersion $-8 \mathrm{ps} / \mathrm{km}-\mathrm{nm}$, the normalized propagation constants (b) at optimized $\mathrm{V}$ parameters are obtained. The optimized value of core index $\mathrm{n}_{1}$ is 1.4529 at $\mathrm{V}$ $=2.14$ and the refractive indices of 1 st clad, $2^{\text {nd }}$ clad and outer clad are given by $1.4462,1.4445$, 1.4435 respectively and the central core radius (a) is given by $3.788 \mu \mathrm{m}$. Similarly, the V parameter corresponding to pump power at wavelength $980 \mathrm{~nm}$ is 3.3912 . Corresponding to the above $\mathrm{V}$ values the numerical solution of the scalar wave equation yields the normalized propagation constant (b) which is defined as

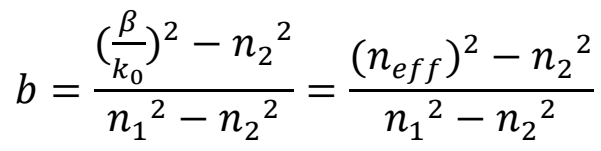

Where $\mathrm{n}_{\text {eff }}$ represents the effective refractive index in the fiber. In order to model the EDFA with the proposed refractive index profile, the actual transverse intensity profiles corresponding to signal and pump are termed as $\left[f_{s}(r)\right]$ and $\left[f_{p}(r)\right]$, which are obtained by solving the scalar wave equation. The so obtained intensity profiles are depicted in Fig.2 and Fig.3.

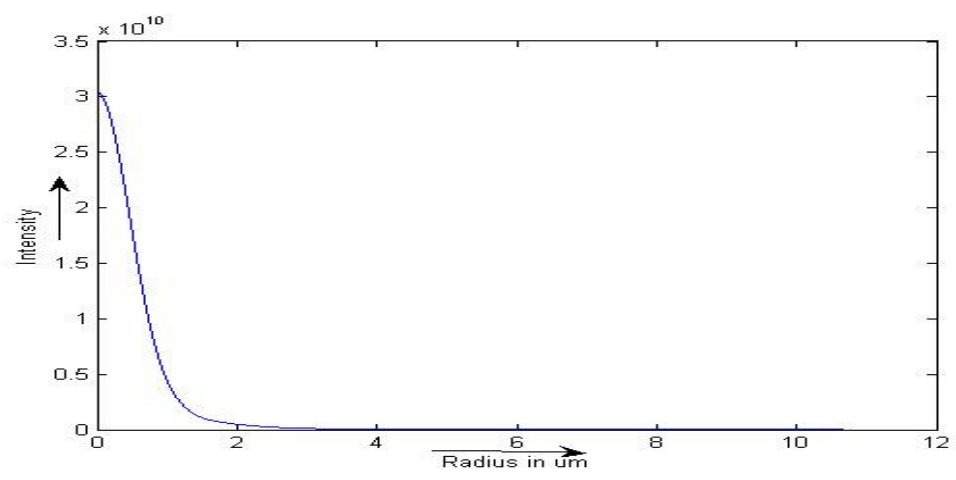

Fig: 2. Transverse intensity distribution for signal wave $\left[f_{s}(r)\right]$

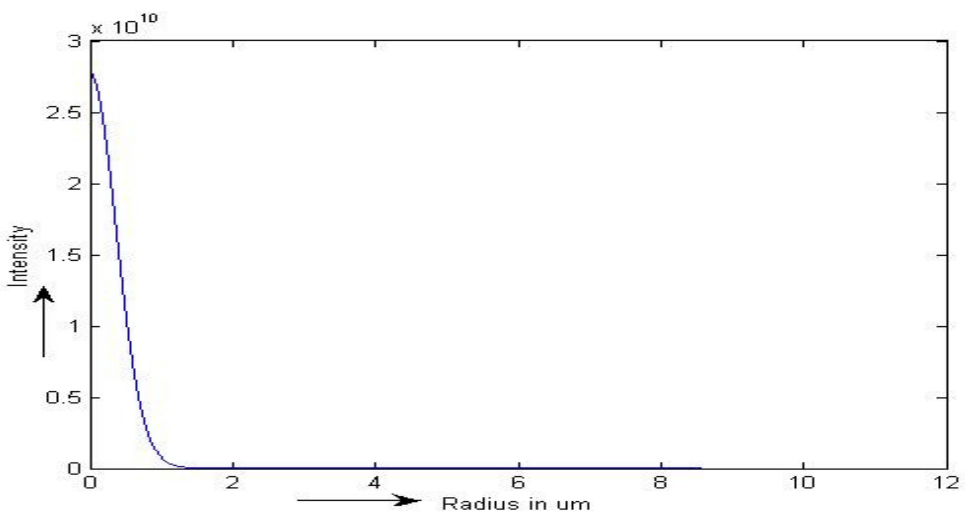

Fig: 3.Transverse intensity distribution for pump wave $\left[f_{p}(r)\right]$ 
Fig 4.Shows the variation of pump power with the length of the fiber for input pump power levels of $20 \mathrm{~mW}$. As expected, the pump power monotonically reduces as it propagates along the fiber. It is worthwhile to note that the decrease is initially almost linear when the power is high and becomes exponential for lower pump powers.

Fig 5.Shows the variation of signal power with fiber length for an input signal power of $\mathrm{P}_{\mathrm{s}}(0)$ $=1 \mu \mathrm{W}$. The signal power increases initially and reaches a maximum value before beginning to reduce. This behavior can be easily understood from the fact that, as the pump light propagates through the fiber, it gets absorbed by the fiber and the pump level keeps falling monotonically with fiber length $(\mathrm{z})$. Thus, the inversion keeps reducing with $\mathrm{z}$, which in turn reduces the gain at any value of $\mathrm{z}$. At some $\mathrm{z}$ value the pump power drops below the critical value, wherein the fiber becomes attenuating rather than amplifying.

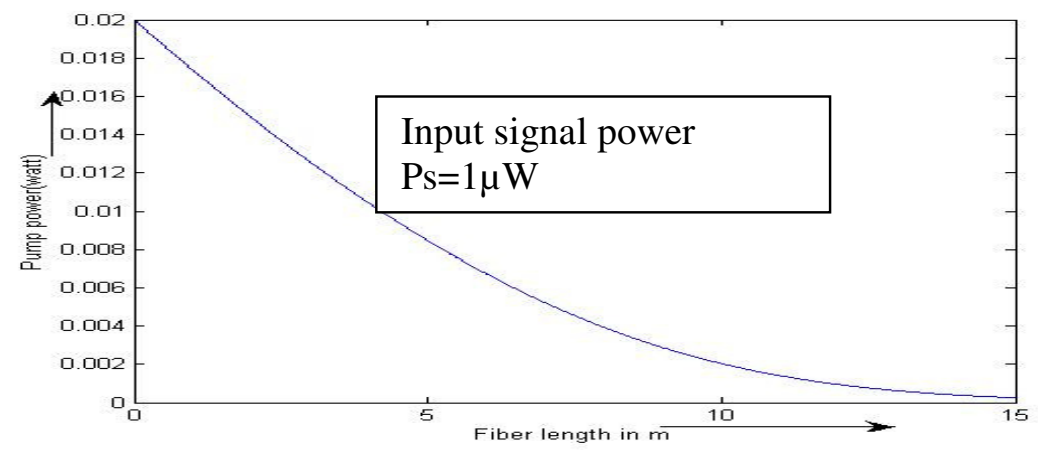

Fig: 4.Variation of pump power with fiber length



Fig: 5.Variation of signal power with fiber length

Solving theseequations for the lengthwise variations of pump and signal power is obtained. Fig. 6 shows the variation of signal power with length for an input signal power $\left(\mathrm{P}_{\mathrm{s}}(0)\right)$ of $1 \mu \mathrm{w}$ and input pump power $\left(P_{p}(0)\right)$ of $30 \mathrm{mw}$. It is seen that the signal power increases initially and reaches a maximum value at fiber length of $9.27 \mathrm{~m}$ before beginning to reduce. The length at which signal power becomes maximum can be called as optimum length. The study also shows that as the pump power increases the optimum length $\left(\mathrm{L}_{\mathrm{opt}}\right)$ of the signal power becomes more. This is due to the fact that more number of erbium atoms can be inverted at higher pump intensity to enable the amplification process.

At the same time Fig. 6 shows the variation of pump power with length of the fiber. The pump power monotonically reduces as it propagates along the fiber. The figures show that for high 
pump power of $30 \mathrm{~mW}$ the decrease is initially almost linear and becomes exponential for low pump power of $20 \mathrm{~mW}$, by following the well known nature of variation i.e., $e^{-\alpha_{p} z}$.

The figure shows the variation of gain with length of the fiber. The figures showsthat for every input pump power level there is an optimum length of the fiber for achieving maximum gain. For example when input pump power $\operatorname{Pp}(0)=30 \mathrm{mw}$ the optimum length is $9.27 \mathrm{~m}$. The optimum length arises because, as the pump power propagates through the fiber, it gets absorbed by the fiber and the pump power decreases according to the $e^{-\alpha_{p} z}$, where $\alpha_{\mathrm{p}}$ is the absorption coefficient corresponding to pumpwavelength. Thus the inversion keeps reducing with $\mathrm{z}$ which in turn reduces the gain. When the pump power decreases below the threshold value, the fibers become attenuating rather amplifying. Beyond the optimum length the amplified signal reabsorbs, which finally reduces the gain.
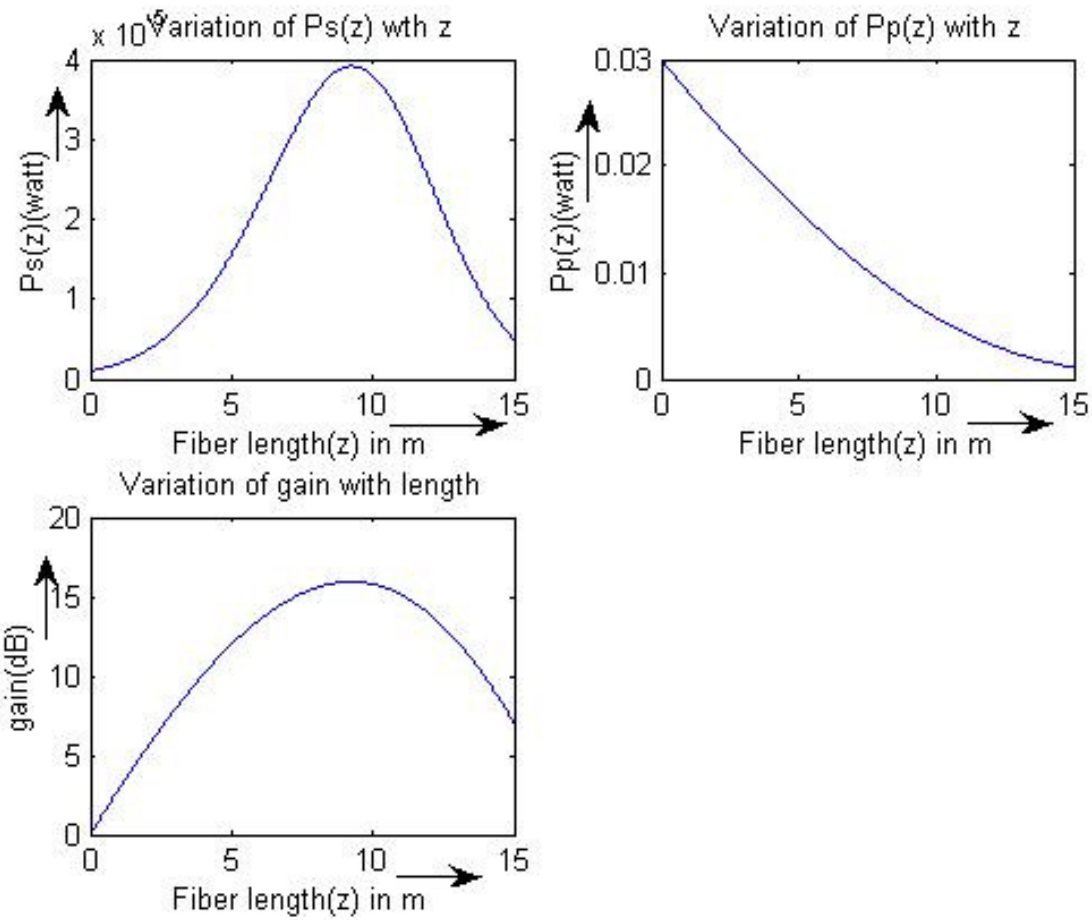

Fig: 6. Variation of (a) signal power, (b) pump power \& (c) gain with length for input signal power $1 \mu \mathrm{W}$ and input pump power $30 \mathrm{~mW}$

Fig.7 shows the variation of the optimum length with input pump power and fig.8 shows the variation of gain with the input pump power for fiber length of $15 \mathrm{~m}$. For any fiber length there is a threshold pump power for transparency of the fiber. At this pump power, the signal gets neither attenuated nor amplified. Beyond this threshold pump power, the gain increases with increasing pump power, finally saturating at large pump powers. The saturation behavior is essentially due to the fact that as the pump power is increased, more and more erbium ions get inverted. Hence there would be no more increase in inversion and, hence, gain. 

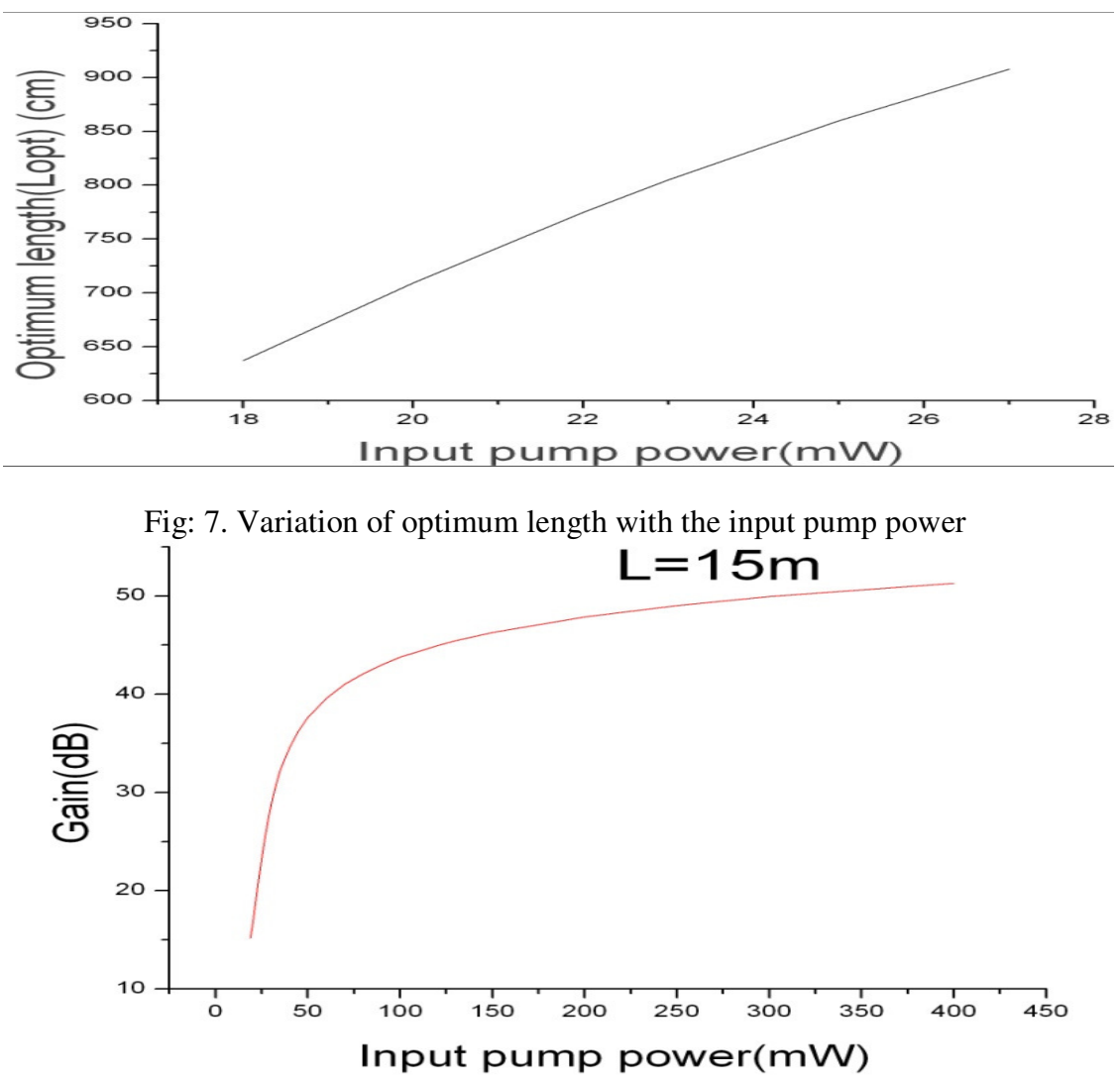

Fig: 8. Variation of gain with input pump power for fiber length $\mathrm{z}=15 \mathrm{~m}$

In fig.9 the variation of optimum length with input pump power is shown for five different input signals with power $1 \mu \mathrm{W}, 5 \mu \mathrm{W}, 7 \mu \mathrm{W}, 10 \mu \mathrm{W} \& 15 \mu \mathrm{W}$ respectively. Fig. 10 shows the variation of maximum gain with input pump power for five different input signal $1 \mu \mathrm{w}, 5 \mu \mathrm{w}, 7 \mu \mathrm{w}, 10 \mu \mathrm{w}$, $15 \mu \mathrm{w}$. From this figure it can be seen that optimum length as well as maximum gain increases with increase in input pump power. This phenomenon happens as the input pump power increases it takes more and more length to decrease up to the optimum condition where attenuation starts to be dominating. Here increase in pump power delay the process of reaching that value. From the fig 9 \& 10 we can also conclude that in small signal power region the maximum gain and $\mathrm{L}_{\text {opt }}$ are more or less independent of input signal power. Differences among the gains and $\mathrm{L}_{\mathrm{opt}}$ for different input signal powers at the same input pump power are distinct when the input pump power value is sufficiently high.

\section{Conclusion}

A dispersion compensating fiber amplifier is designed and optimized in the present work and the amplifier is modeled to obtain its characteristics. The gain profile of the Erbium doped DCF amplifier for different sets of input pump power and signal power are obtained. Length wise variation of input pump power and input signal power are presented in this work. The study shows that optimum length increases with increase in input pump power when the input signal power is a constant parameter. On the other hand, for a particular input pump power as the input signal power increases the optimum length decreases. For different signal power of Erbium doped DCF, the modeling shows that maximum gain and optimum length areal most linear with input 
pump power. This study may be useful for fiber optics manufactures to fabricate an Erbium doped DCF amplifier.
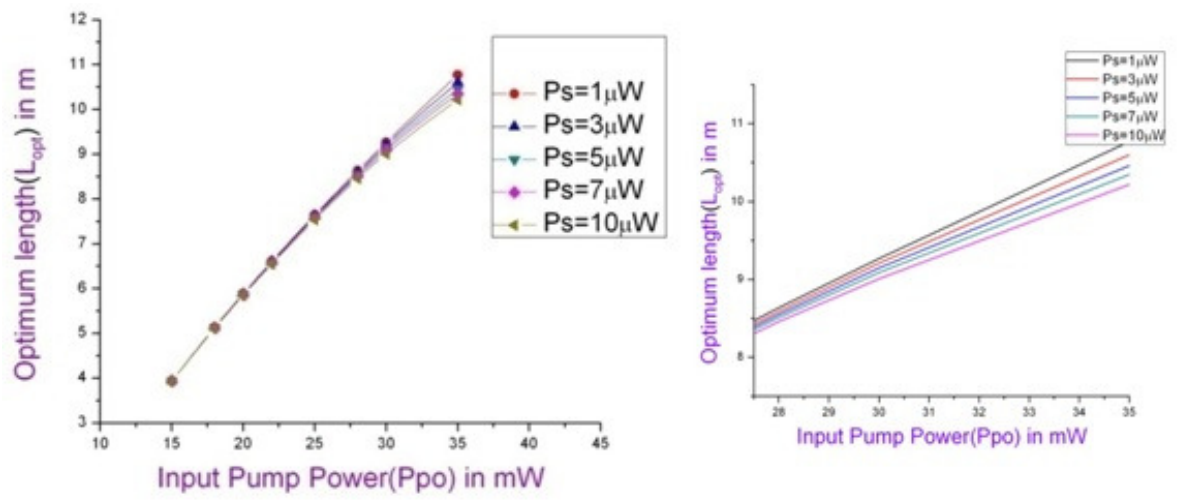

Fig: 9. Variation of optimum length with input pump power for differentinput signal power
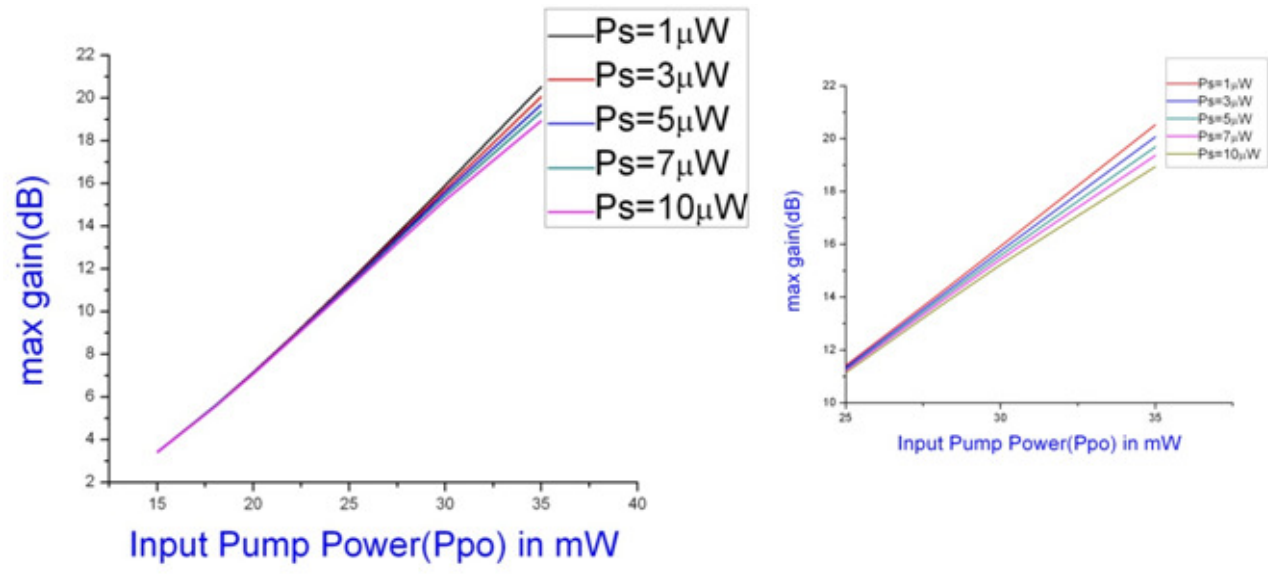

Fig: 10. Variation of maximum gain with input pump power for different signal power

\section{REFERENCES}

[1] Ghatak and Thyagarajan.," Introduction to fiber optics”. Cambridge University Press, 1999.

[2] Izadpanah, H., Lin,C., Gimlett, J.L., Antos, A.J., Hall, D. W. and Smith, D.K., “ Dispersion compensation in 1310nm optimized SMFs using optical equalizer fiber, EDFAs and 1310/1550 nm WDM ", Electron. Lett., 28 ,1469 (1992).

[3] R.J.mears, Reekie, I.m.jauncey, and D.N.payne,"'low noice erbium doped fiber amplifier operating at $1.54 \mu \mathrm{m}$,'Electron .Lett.,vol.23,1987.

[4] E. Desurvire, "Erbium doped Fiber Amplifier Principle and Applications", p.29,Wiley-Interscience, New York.1994.Photo

[5] Giles C.R,. Desurvire E., Talman, J.R., Simpson J.R. \& Becker P.C., "2-Gbit/sec signal and amplification at $=\lambda(1.53 \mathrm{um})$ in an erbium doped single- mode fiber amplifier, " J.Light wave technol.,no1.,vol.7, 1989.

[6] Cheng, C. and M. Xiao, "Optimization of an erbium-doped fiberamplifier with radial effects," Opt. Commun., Vol. 254, 215-222, 2005.

[7] Cheng, C., "A global design of an erbium-doped fiber and an erbium-doped fiber amplifier," Opt. LaserT echnol., Vol. 36, 607-612, 2004. 
[8] Antos, A. J., Hall, D. W. and Smith, D.K., "Dispersion compensating fibre for upgrading existing $1310 \mathrm{~nm}$ optimized systems to $1550 \mathrm{~nm}$ operation", OFC/IOOC, 204(1993).

[9] Basu, Tewari, R., Acharya, H. N., "Effect of grading on the characteristics of a dispersion compensated fiber”, Optics Column., 174, 119(2000).

\section{AUTHORS}

Mithun De has received his M.Sc degree in Physics from Bengal Engineering and Science University, Shibpur. (W.B). He is now working as an assistant professor in GIMT .Krishnagar Nadia

Indranil Mondal has received his M.Sc degree in Physics from Guru Ghasidas University,Bilaspur (C.G).He is now workingas an assistant professor in GIMT. KrishnagarNadia

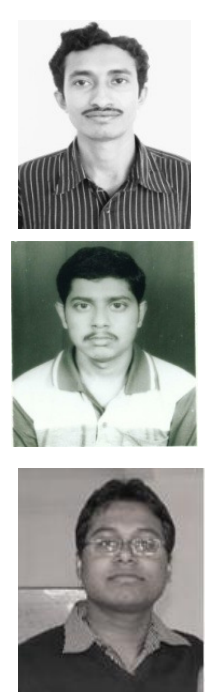

Amit Kumar Das has received his M.Sc degree in Physics from Guru Ghasidas University,Bilaspur (C.G).He is now working as an assistant professor in GIMT. Krishnagar,Nadia 